\title{
UN ESTUDIO SOBRE LA CONDICION DE LA ALMEJA PISMO Tivela stultorum CON DATOS DE LONGITUD Y PESO DE LA CARNE DE CAPTURAS COMERCIALES
}

\author{
por \\ Ricardo Searcy Bernal \\ Instituto de Investigaciones Oceanológicas \\ Universidad Autónoma de Baja California \\ Apartado Postal No. 453 \\ Ensenada, Baja California, México
}

SEARCY BERNAL, R., 1983. Un estudio sobre la condición de la almeja Pismo tivela stultorum con datos de longitud y peso de la carne de capturas comerciales. Ciencias Marinas, 9 (2): 19-30.

\section{RESUMEN}

Se desarrolla un índice parà medir la condición en bivalvos, basado en datos de longitud de la conctia y peso de la carne, aplicándose para la almeja pismo de la Playa San Ramón, B. C. México. Este índicc o peso estándar, es el peso húmedo de la carne estimado para una longitud fija, mediante el uso de regresiones funcionales de media geométrica entre ambas variables.

Las fluctuaciones del peso estándar se asocian con los principales cambios en la actividad reproductiva de esta especie durante un ciclo anual, con excepción del período de mayor precipitación pluvial.

Este índice puede ser una alternativa para inferir mayor información biológica de estadísticas pesqueras en otros recursos acuáticos.

\section{ABSTRACT}

A condition index for bivalves is developed based on shell length and meat weight data and is applied to pismo clams from Playa San Ramón, B. C., México. This index or standard weight, is the estimated wet meat weight for a fixed lenght, using the geometric mean functional regression between these two variables.

Standard weight fluctuations can be associated with the main changes in reproductive activity during the annual cycle, with the exception of the heaviest rain period.

This index could be an alternative for infering more biological information from catch statistics of other aquatic resources.

\section{INTRODUCCION}

La condición en bivalvos se ha utilizado tradicionalmente tanto con fines comerciales como biológicos (Baird, 1958;
Walne, 1970). Aunque no existe una definición generalizada. podemos entender la condición como el grado de "gordura" que presenta el organismo del que se trate. 
Desde el punto de vista comercial, esta característica se asocia directamente con el rendimiento del producto y en algunos casos, con la mayor preferencia del consumidor por el sabor y consistencia que caracterizan a organismos con condición alta. En este sentido, puede usarse para valorar la calidad del producto según alguna escala predeterminada, o para compararla en distintas épocas del año, localidades o condiciones ambientales (Walne, 1970; Gabbot y Walker, 1971).

En términos biológicos, las fluctuaciones de la condición de un bivalvo se asocian generalmente con su estado reproductivo o nutricional; siendo particularmente importante la posibilidad de hacer inferencias generales sobre el ciclo reproductivo de una especie, en base a mediciones de la condición. (Baird, 1958; Hickman e Illingworth, 1980; Santiago y Rojas, 1982).

Se han urilizado diferentes índices para medir la condición de un bivalvo. Posiblemente el más conocido es el descrito por Baird (1958), que consiste en el porcentaje de la cavidad intervalvar que es ocupado por carne. Su determinación requiere de la medición de los volúmenes totales de valvas y carne, por desplazamiento directo de agua, después de una cuidadosa disección.

Otros autores han propuesto usar índices que utilizan peso húmedo en lugar de volumen; peso seco en lugar de peso húmedo; o bien el contenido de algún componente bioquímico como glucógeno, según los objetivos y precisión deseados en cada investigación. Discusiones amplias sobre estos distintos enfoques se pueden consultar en Walne (1970) y Kickman e Illingworth (1980) y quedan fuera del propósito de este trabajo.

Una característica común de todos esos métodos, es que requieren procedimientos tardados y que generalmente deben ocupar un esfuerzo de toma de muestras y trabajo de laboratorio especial, dentro de los programas de investigación sobre recursos pesqueros, que no frecuentemente pueden ser financiados en países como el nuestro, donde el presupuesto para el desarrollo científico es moderado.

Por lo general, cualquier programa de investigación pesquera en México incluye, como una parte fundamental y a veces única, la toma de muestras de capturas comerciales con el registro de longitudes, pesos y edades, ya que es la forma más barata y accęsible de obtener información biológica sobre un recurso. Vale la pena entonces, hacer esfuerzos para optimizar el uso de esos datos.

La almeja pismo Tivela stultorum es uno de los invertebrados marinos de mayor importancia comercial en Baja California (Tabla 1). Este recurso fue objeto de una investigación multidisciplinaria por el Instituto de Investigaciones Oceanológicas de la UABC, en el período 1978-1981, que incluyó registros de estadísticas pesqueras y estudios poblacionales diversos.

El propósito de este trabajo es derivar algún índice de condición que pudiera usarse con fines biológicos, utilizando colateralmente los datos de longitud y peso de la carne, obtenidos como parte de la toma periódica de muestras de capturas comer-

TABLA 1. Volúmenes de producción de los principales invertebrados marinos registrados en Baja California (kg) durante 1977 y 1978.

\begin{tabular}{lcc}
\hline PRODUCTO & 1977 & 1978 \\
\hline ALMEJA PISMO & 1 '076,802 & 1 '520,988 \\
MEJILLON & 402,484 & 200,538 \\
ERIZO & 231,156 & 260,235 \\
CALAMAR & 353,046 & 36,882 \\
LANGOSTA & 130,964 & 134,199 \\
ABULON & 124,965 & 87,983
\end{tabular}

Fuente: Secretaria de Pesca, Ensenada, B. C.

El propósito de este trabajo es derivar algún índice de condición que pudiera usarse con fines biológicos, utilizando colateralmente los datos de longitud y peso de la carne, obtenidos como parte de la toma periódica de muestras de capturas comer- 
ciales de almeja pismo que se desarrolló en dicho proyecto (Searcy, 1982).

\section{MATERIALES Y METODOS}

Se colectaron 22 muestras aleatorias de las capturas comerciales de almeja pismo extraídas en Playa San Ramón, B. C. (Figura 1), principal árca almejera del estado, en el lapso comprendido entre julio de 1978 y marzo de 1980 , con periodicidad aproximada de un mes. El tamaño de muestra fue fijado durante 1978 en el $1 \%$ de la captura del día y posteriormente se determinó un número de 62 almejas, que se trató de respetar como muestra mínima según el método descrito por Sokal y Rohlf (1981), para una $\alpha=0.05$ y $P=99 \%$, con referencia al análisis de las tallas de las muestras iniciales (Searcy, 1982). A cada organismo se le determinó la talla (longitud antero posterior), peso de la carne, peso total y peso de las valvas utilizando materiales convencionales. Se determinó el sexo mediante la observación microscópica de un frotis gonadal, y se computó la proporción de sexos y de organismos indiferenciados para cada muestra, con excepción de las dos primeras.

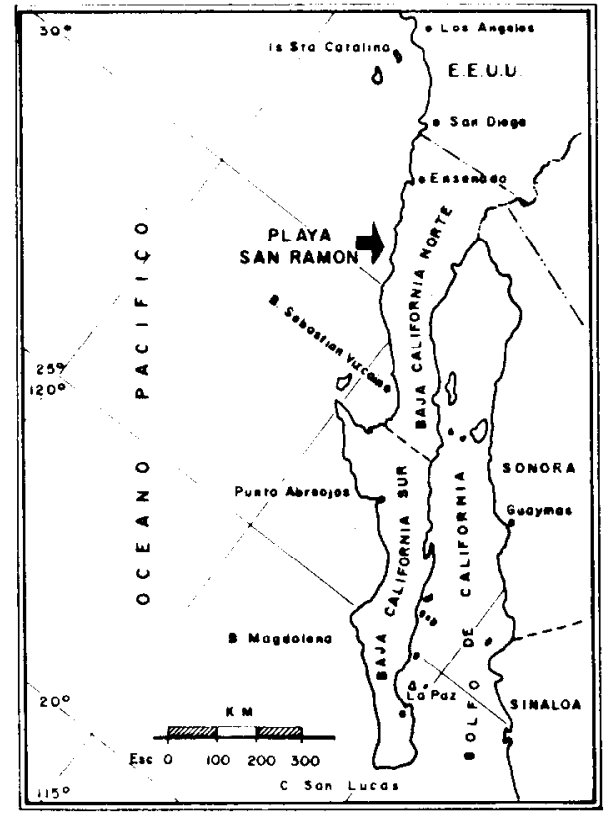

FIGURA 1. Localización de la zona de estudios.
Para decidir el tipo de análisis estadístico a utilizar (paramétrico o no paramétrico), se realizaron pruebas de bondad de ajuste a la distribución normal para los datos de longitud y peso de la carne, según el método de Kolmogorov-Smirnov (Sokal y Rohlf, 1981). Se probó además la homogeneidad de varianzas entre las muestras de cada tipo, por el método de Bartlett (Snedecor y Cochran, 1967).

Con el propósito de probar la validez de la combinación de los datos de machos y hembras, se practicaron pruebas $t$ para tallas $y$ pesos en tres muestras seleccionadas en la época de madurez sexual.

Si se puede asumir que la forma del bilvalvo no varía en el rango de longitudes considerado, y si además es válido suponer que el espesor de la concha guarda una relación lineal respecto a la longitud, se puede sugerir entonces que la diferencia en el peso de la carne de dos almejas de exactamente la misma longitud, refleja una diferencia en la condición. De manera que los datos de peso de la carne, normalizados respecto a la longitud, podrían ser usados como un índice de condición.

Para realizar esa normalización, se estimaron los parámetros de las regresiones logarítmicas funcionales de media geométrica (GM) (Ricker, 1973) entre longitud y peso de la carne para cada muestra, y posteriormente el peso esperado para una longitud constante, equivalente a la media global de todas las muestras $(12.2 \mathrm{~cm})$. El peso estándar así calculado, es entonces, el índice de condición utilizado en este trabajo.

La regresión GM produce estimaciones menos sesgadas de los parámetros poblacionales de la regresión, en comparación con los métodos tradicionales, cuando se cuenta únicamente con tallas en el rango comercial (Ricker, 1973). Para probar si ésto ocurría en nuestro caso, se colectó una muestra en septiembre de 1980 en la zona de estudio, incluyendo todo el rango poblacional de tallas, y se procesó por ambos métodos regresivos.

Con el propósito de examinar la rela- 
ción entre el peso estándar y la actividad reproductiva, se utilizó el porcentaje de almejas a las que puede determinársele el sexo en cada muestra (P.A.S.); es decir, la proporción de organismos que presentaban productos sexuales identificables en los frotis gonadales, sin importar su estadio de gametogénesis. Adicionalmente, se hicieron algunas observaciones cualitativas sobre el estado aparente de turgencia de las gónadas.

Para explicar algunas variaciones en el peso estándar, se utilizaron datos oficiales sobre precipitación pluvial en la zona; así como información sobre la composición bioquímica del bivalvo dentro del período con- siderado (Macias et al., 1979).

Finalmente, se realizó un análisis de correlación simple y múltiple, usando técnicas no paramétricas (Spearman) en combinación con métodos paramétricos (Conover, 1980; Sokal y Rohlf, 1981), para examinar las relaciones entre las tres variables: peso standard, P.A.S. y volumen promedio de precipitación. La última de éstas, se utilizó con un desfase de un mes, por consideraciones que se comentarán en la sección apropiada.

\section{RESULTADOS}

La tabla 2 resume las estimaciones

TABLA 2. Estadísticas básicas sobre la distribución de tallas y pesos de la carne en muestras de captura comercial de almeja Pismo.

\begin{tabular}{|c|c|c|c|c|c|c|c|}
\hline \multirow{2}{*}{$\begin{array}{l}\text { Fecha } \\
\text { Muestreo }\end{array}$} & \multirow[b]{2}{*}{$\mathbf{n}$} & \multicolumn{3}{|c|}{ Tallas $(\mathrm{cm})$} & \multicolumn{3}{|c|}{ Peso carne (gr) } \\
\hline & & $\overline{\mathbf{X}}$ & $\mathbf{S}$ & D & $\overline{\mathbf{X}}$ & $\mathbf{S}$ & $\overline{\mathbf{D}}$ \\
\hline $\mathrm{JL} / 08 / 78$ & 104 & 12.31 & 0.91 & 0.05868 & 72.65 & 14.04 & 0.05893 \\
\hline $\mathrm{JL} / 21 / 78$ & 130 & 12.21 & 0.87 & 0.10941 & 68.28 & 13.70 & 0.09450 \\
\hline $\mathrm{AG} / 01 / 78$ & 78 & 11.98 & 0.78 & 0.09703 & 69.24 & 13.73 & 0.14730 \\
\hline $\mathrm{AG} / 22 / 78$ & 78 & $11: 97$ & 0.89 & 0.10623 & 65.12 & 13.91 & 0.08339 \\
\hline $\mathrm{AG} / 29 / 78$ & 139 & 12.20 & 0.94 & 0.10022 & 70.28 & 12.82 & 0.07417 \\
\hline $\mathrm{OC} / 29 / 78$ & 120 & 11.98 & 0.88 & 0.08549 & 60.23 & 11.23 & 0.09130 \\
\hline$N V / 18 / 78$ & 90 & 12.48 & 0.99 & 0.10245 & 63.14 & 13.75 & 0.12731 \\
\hline DC/04/78 & 84 & 12.36 & 0.93 & 0.09573 & 60.50 & 12.78 & 0.08851 \\
\hline DC/29/78 & 62 & 12.46 & 0.96 & 0.06522 & 61.77 & 14.24 & 0.09634 \\
\hline $\mathrm{FB} / 07 / 79$ & 89 & 12.09 & 0.86 & 0.12759 & 65.23 & 14.52 & 0.09159 \\
\hline $\mathrm{MR} / 01 / 79$ & 60 & 12.32 & 0.94 & 0.08653 & 62.87 & 12.75 & 0.06979 \\
\hline $\mathrm{MR} / 15 / 79$ & 72 & 12.62 & 0.97 & 0.08227 & 69.46 & 15.60 & 0.10200 \\
\hline MY/19/79 & 95 & 12.22 & 0.97 & 0.09650 & 69.45 & 14.76 & 0.08450 \\
\hline $\mathrm{JN} / 25 / 79$ & 47 & 12.15 & 0.98 & 0.15913 & 70.78 & 15.64 & 0.15077 \\
\hline $\mathrm{JL} / 24 / 79$ & 58 & 11.75 & 0.87 & 0.05913 & 68.10 & 13.44 & 0.07700 \\
\hline $\mathrm{AG} / 09 / 79$ & 69 & 12.11 & 0.85 & 0.08700 & 74.16 & 15.13 & 0.12474 \\
\hline $\mathrm{SP} / 07 / 79$ & 70 & 11.93 & 0.86 & 0.14187 & 66.24 & 13.66 & 0.11530 \\
\hline $\mathrm{OC} / 07 / 79$ & 74 & 11.93 & 0.87 & 0.11444 & 68.39 & 15.65 & 0.08983 \\
\hline NV/07/79 & 24 & 12.44 & 0.85 & 0.10622 & $\cdots-$ & -..-- & -- \\
\hline $\mathrm{DC} / 18 / 79$ & 72 & 12.47 & 0.94 & 0.10558 & 69.56 & 14.20 & 0.13695 \\
\hline EN/19/80 & 70 & 12.05 & 1.04 & 0.11488 & 60.59 & 16.66 & 0.12432 \\
\hline $\mathrm{MR} / 16 / 80$ & 71 & 11.66 & 0.73 & 0.13600 & 60.71 & 10.96 & 0.10894 \\
\hline
\end{tabular}




\section{Searcy Bernal}

estadísticas básicas de los datos obtenidos. Ningún valor del estadígrafo de Kolmogorov Smirnov (D) es significativo $\left(p_{2} \geq 0.05\right)$. Por otro lado, los valores de $\mathrm{X}^{2}$ para la prueba de Bartlett son 10.78 ( $p>0.9)$ para longitudes y 26.40 ( $p>0.1$ ) para pesos de carne, de manera que se puede justificar el uso de los métodos paramétricos mencionados anteriormente.

En la figura 2 se señalan las variaciones en la longitud y peso medios, observándose que tienden a presentar patrones opuestos que destacan la importancia de la normalización.

La proporción en las muestras de machos a hembras, fluctuó entre 0.35 y 1.40 ; sin embargo, los cesultados de la tabla 3 indican que no existen diferencias significativas entre las longitudes y pesos de ambos sexos. Por lo tanto, se sugiere adecuado el análisis conjunto de esta información.
Las relaciones longitud-peso de la carne utilizando las regresiones predictiva y GM, para la muestra colectada en septiembre de 1980 , se señalan en la Figura 3, notándose la conveniencia de la segunda para inferir la relación poblacional, cuando se usan longitudes del rango comercial únicamente.

Los parámetros estimados de las regresiones GM entre ambas variables se señalan en la Tabla 4, incluyendo las estimaciones de peso estándar. Todos los coeficientes paramétricos de correlación fueron significativos $(P>0.01)$.

El comportamiento del peso estándar y del porcentaje de almejas a las que puede determinársele el sexo, se indica en la Figura 4. Durante 1978, el drenado de la carne antes de la determinación del peso era mayor que en la rutina adoptada posteriormente.

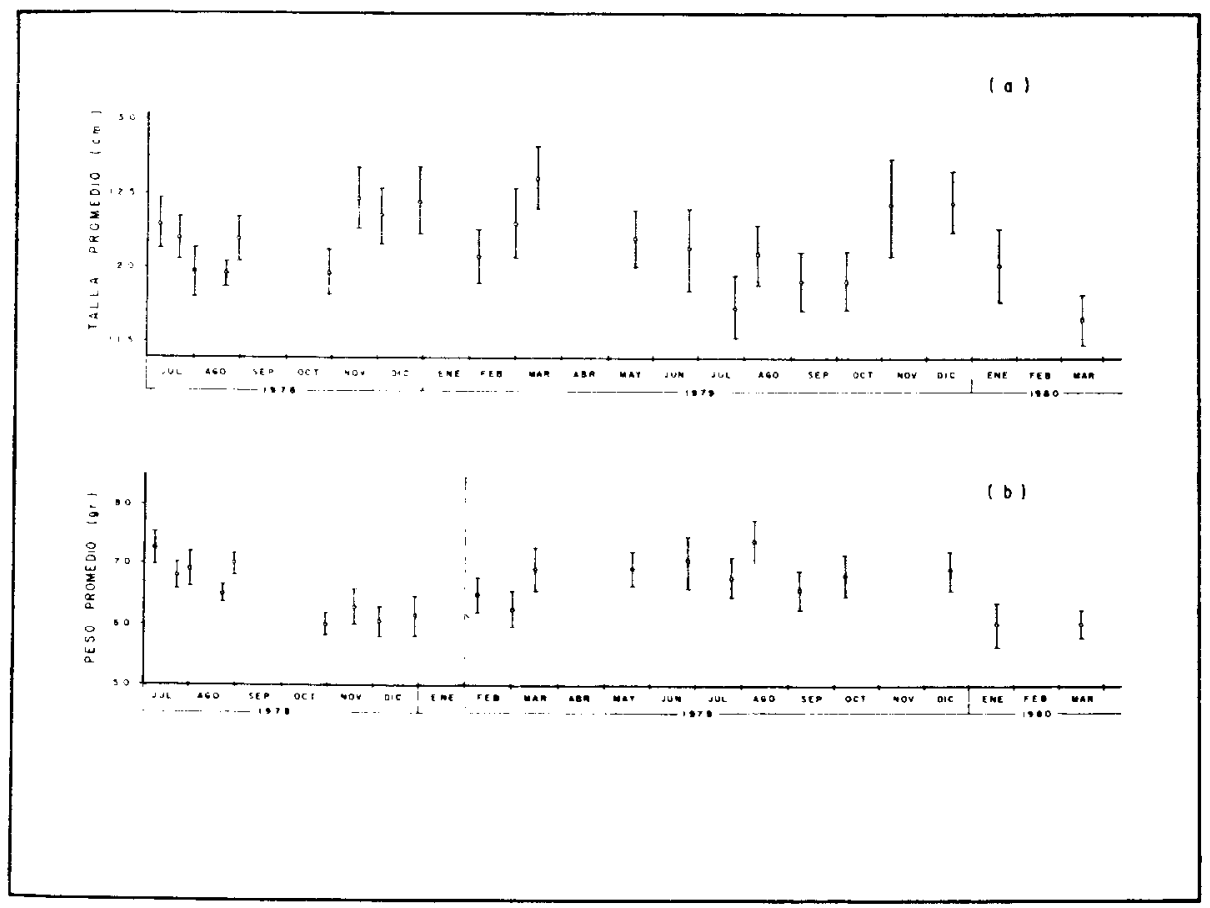

FIGURA 2. Variación de la talla promedio (a) y el peso promedio de la carne (b) en las muestras de captura comercial de almeja Pismo ( $T$. stultorum) a lo largo del período de estudio.

Las barras verticales señalan intervalos de confianza al $95 \%$ y la línea punteada, cambios en la metodologia de pesado (ver texto). 


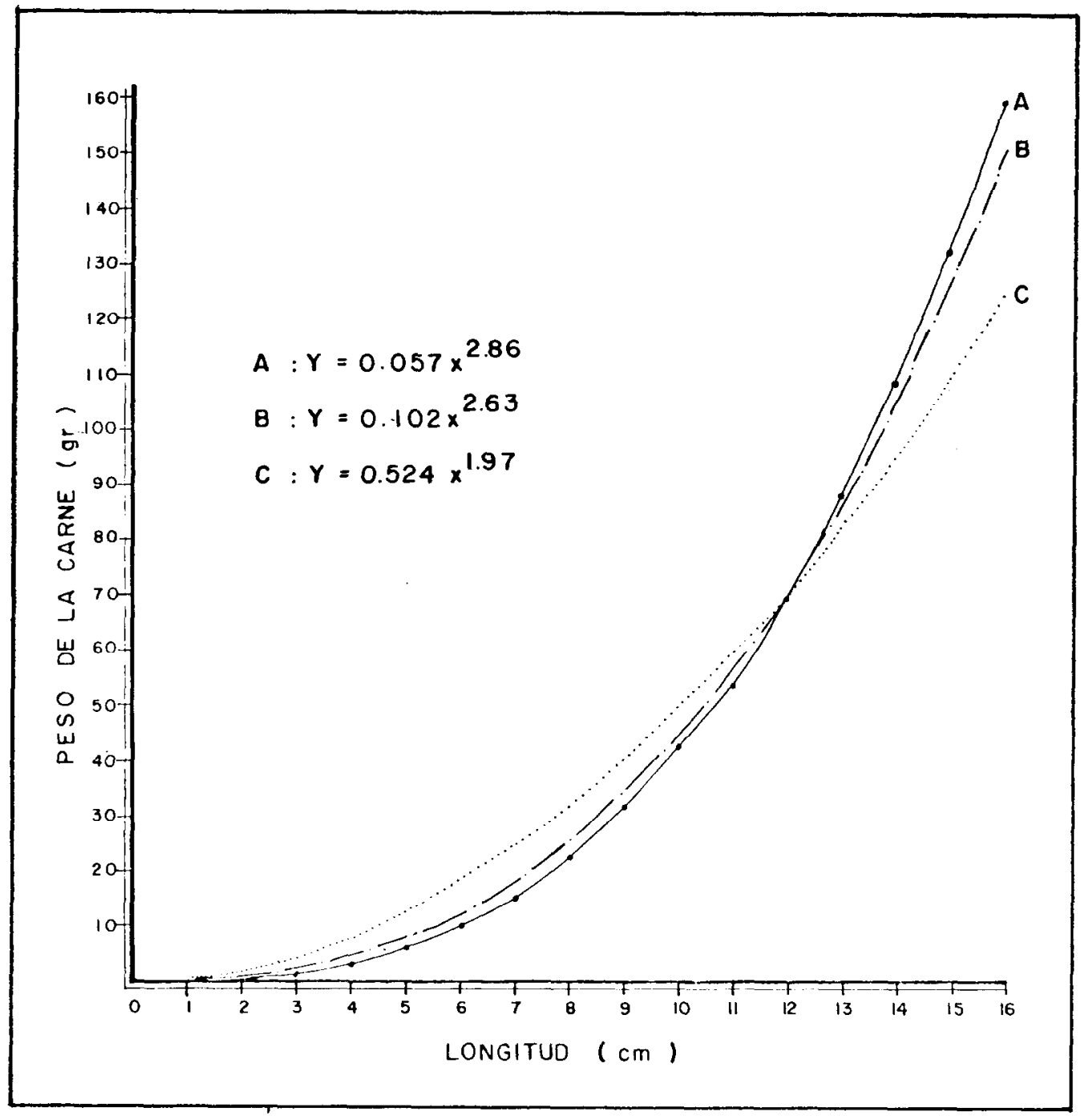

FIGURA 3. Relación longitud-peso para una muestra colectada en septiembre de 1980. La curva A representa las regresiones predictiva y funcional $(\mathrm{GM})$, considerando un rango amplio de tallas. Las curvas $B$ C representan las regresiones GM y predictiva respectivamente aplicadas a tallas comerciales.

Por este motivo, los valores de peso estándar para ese año son ligeramente menores quc los que corresponderían a la nueva rutina.

Se puede observar que las curvas de peso estándar y P.A.S. siguen un patrón cíclico similar, con dos discordancias principales en los meses de octubre a diciembre de
1978, por un lado, y los meses de febrero de 1979 y marzo de 1980 , por el otro (Figuras 4a y $4 b$ ).

La Figura 5 reporta los volúmenes de precipitación mensual promedio en la región, y se puede observar que las discordancias de febrero de 1979 y marzo de 1980 en la Figu- 
Searcy Bernal

TABLA 3. Resumen de los resultados de las pruebas $t$ para tallas y pesos de machos y hembras, en tres muestras del verano de 1979.

\begin{tabular}{llllll}
\hline \multirow{2}{*}{ Muestras } & & \multicolumn{2}{c}{ Tallas } & & \multicolumn{2}{c}{ Peso carne } \\
\cline { 3 - 6 } & g.1 & $\mathbf{t}$ & $\mathbf{P}$ & & $\mathbf{t}$ \\
\hline $\mathrm{JN} / 25 / 79$ & 42 & 0.601 & 0.55 & 0.812 & 0.42 \\
$\mathrm{JL} / 24 / 79$ & 54 & 0.563 & 0.57 & 0.794 & 0.43 \\
$\mathrm{AG} / 09 / 79$ & 65 & 0.291 & 0.77 & 0.129 & 0.89 \\
\hline
\end{tabular}

TABLA 4. Parámetros de las regresiones GM (1) entre la talla y el peso de la carne de cada muestra analizada.

\begin{tabular}{lllll}
\hline $\begin{array}{l}\text { FECHA DE } \\
\text { MUEST RO }\end{array}$ & $\mathbf{v}$ & $\mathbf{a}$ & $\mathbf{S}$ & $\begin{array}{c}\text { P } \\
\text { std } \\
\text { (gr) }\end{array}$ \\
\hline JL/08/78 & & & $\mathbf{v}$ & 70.15 \\
JL/21/78 & 2.612 & 0.102 & 0.163 & 67.19 \\
AG/01/78 & 2.818 & 0.058 & 0.178 & 72.24 \\
AG/22/78 & 3.068 & 0.034 & 0.272 & 67.88 \\
AG/29/78 & 2.948 & 0.042 & 0.265 & 69.55 \\
OC/29/78 & 2.413 & 0.166 & 0.138 & 62.30 \\
NV/18/78 & 2.494 & 0.121 & 0.135 & 58.56 \\
DC/04/78 & 2.645 & 0.078 & 0.158 & 57.58 \\
DC/29/78 & 2.766 & 0.057 & 0.158 & 57.15 \\
FB/07/79 & 2.860 & 0.045 & 0.235 & 65.95 \\
MR/01/79 & 3.035 & 0.033 & 0.189 & 60.38 \\
MR/15/79 & 2.781 & 0.057 & 0.238 & 61.99 \\
MY/19/79 & 2.887 & 0.045 & 0.196 & 71.06 \\
JN/25/79 & 2.756 & 0.069 & 0.227 & 74.69 \\
JL/24/79 & 2.868 & 0.054 & 0.272 & 74.68 \\
AG/09/79 & 2.816 & 0.065 & 0.237 & 69.53 \\
SP/07/79 & 2.948 & 0.047 & 0.277 & 72.26 \\
OC/07/79 & 2.775 & 0.067 & 0.199 & 72.95 \\
NV/07/79 & 3.266 & 0.020 & 0.298 & 64.81 \\
DC/18/79 & 2.705 & 0.084 & 0.336 & 61.54 \\
EN/19/80 & 2.677 & 0.080 & 0.189 & 68.23 \\
MR/16/80 & 3.200 & 0.020 & 0.236 & \\
\hline
\end{tabular}

(1) Según el modelo $\ln Y=\ln a+v \ln X$; donde $Y=$ peso; $X=$ talla; $v=$ pendiente; ln a $=$ ordenada al origen. $S_{\mathrm{V}}$ es la desviación estándar de la pendiente y $\mathrm{P}_{\text {std }}$ es el peso estándar estimado para una almeja de $12.2 \mathrm{~cm}$. 


\section{Searcy Bernal}

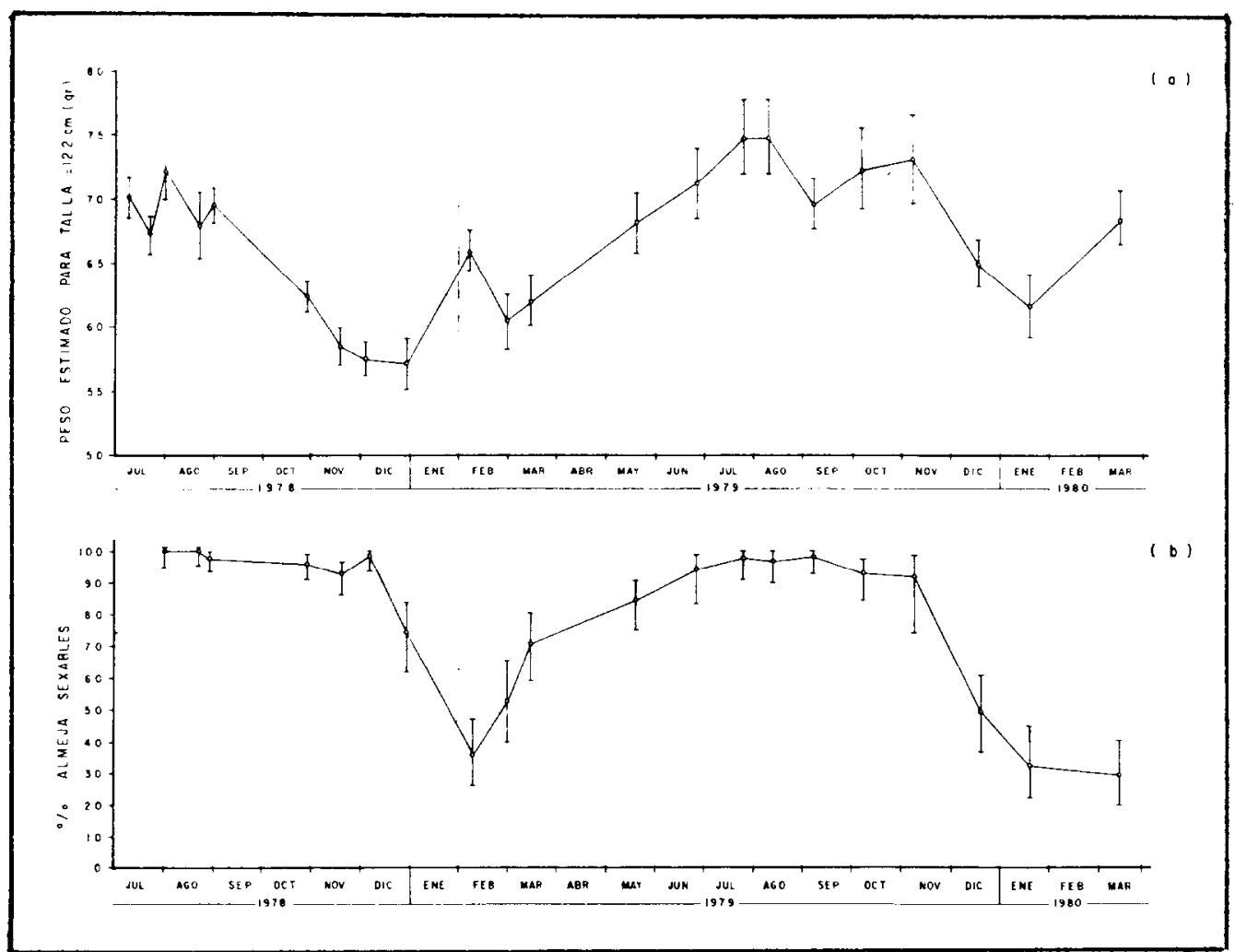

FIGURA 4. Variación del peso húmedo promedio estimado para almejas de talla media $(12.2 \mathrm{~cm})(\mathrm{a})$ y del porcentaje de organismos a los que se les puede determinar sexo (b) a lo largo del período de estudio. Las barras verticales marcan los límites de confianza al $95 \%$ y la línea punteada señala cambios en la metodología de pesado (ver texto).

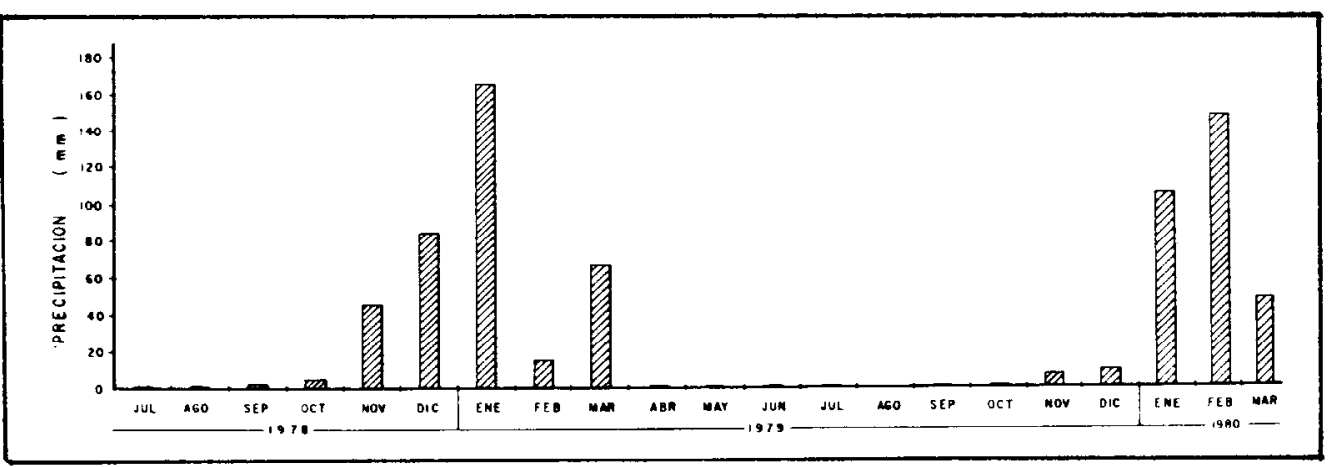

FIGURA 5. Precipitación mensual promedio $(\mathrm{mm})$ de tres estaciones hidrométricas cercanas a Playa San Ramón (Santa María, Las Escobas y colonia Guerrero). 
ra 4, ocurren un mes después de los máximos de precipitación en cada ciclo. La Figura 6 señala la variación del contenido de agua y cenizas en homogeneizados del contenido de la cavidad intervalvar, incluyendo tejidos y fluidos corporales y extra corporales (Macías et al., 1979).

Por último, en la Tabla 5 se resumen los resultados del análisis de correlación entre las tres variables consideradas, con los datos de precipitación de un mes anterior y habiendo corregido las estimaciones de peso standard de 1978 por el cambio en la metodología de pesado. Se reportan coeficientes para todos los datos disponibles $(n=20)$ y para el grupo resultante al eliminar las tercias de octubre a diciembre de $1978(n=16)$.

TABLA 5. Coeficientes de correlación de Spearman entre las variables peso estándar $(x)$, porcentaje de almejas a las que se les puede determinar el sexo (Y) y volumen de precipitación (z) para dos conjuntos de datos. (ver texto).

\begin{tabular}{|c|c|c|}
\hline Coeficiente & $n=20$ & $n=16$ \\
\hline$D_{x y}$ & $0.499^{*}$ & $0.741 * *$ \\
\hline$\rho_{x z}$ & $0.672 * *$ & 0.548 * \\
\hline${ }^{p} Y_{z}$ & $-0.485^{*}$ & $0.601 *$ \\
\hline$P_{X . Y z}$ & $0.701 * *$ & $0.752 * *$ \\
\hline$\rho_{X . Y Z}$ & $0.491 * *$ & $0.566 * *$ \\
\hline n. $s .=$ no signi & 0.05 & $* *=\mathrm{P} \quad 0.01$ \\
\hline
\end{tabular}

\section{DISCUSION}

a) Comparación del peso estándar con otros índices de condición.

El índice de condición de Baird (1958), se puede expresar como:

$$
I=\frac{\text { volumen de la carne }}{\text { volumen de la cavidad }} \times 100
$$

de manera que se puede entender como una medida del volumen de la carne, normalizada respecto al volumen de la cavidad. El peso estándar, por otro lado, se puede entender como una medida del peso de la carne, normalizada respecto a la longitud.

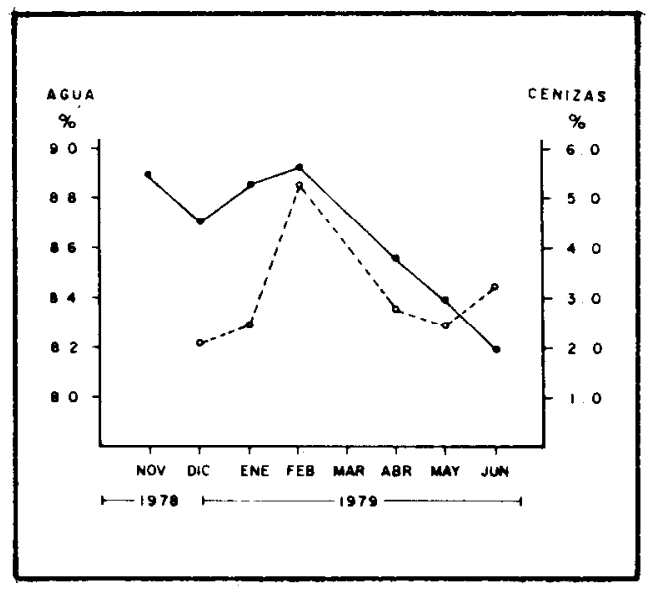

FIGURA 6. Contenido de agua ( ) y cenizas ( ) en almeja Pismo de Playa San Ramón, referidos al peso húmedo (ver texto). (Modificado de Macías et al. 1979).

De modo que si el volumen de la carne se puede asumir proporcional a su peso, y el volumen de la cavidad se puede considerar proporcional a su longitud, ambas medidas de condición deberán ser proporcionales entre sí.

En el primer caso, el problema es mínimo ya que la densidad de la carne es la constante que relaciona su peso y volumen de una manera lineal, de acuerdo a principios físicos básicos.

En el segundo caso, podemos expresar la relación entre el volumen total $\left(V_{t}\right)$ y la longitud $\left(L_{t}\right)$ según el modelo $V_{t}=C_{1} L_{t} b$, donde $\mathrm{C}_{1} \mathrm{y} b$ son constantes.

Ahora bien, si el volumen delimitado por la superficie exterior de las valvas es de la misma forma que el delimitado por la superficie interior, el volumen de la cavidad $\left(V_{i}\right)$ se puede expresar como:

$$
V_{i}=C_{1} L_{i} b=C_{1}\left(L_{t}-2 G\right) b
$$

donde $L_{i}$ es la longitud de la cavidad, que equivale a la longitud total menos el doble del espesor de las valvas (G), asumiendo que éstas son simétricas y regulares.

Si existe además una relación lineal 
entre $G$ y $L_{t}$ a través de una constante $C_{2}$, entonces:

$$
\begin{aligned}
& V_{i}=C_{l}\left(L_{t}-2 C_{2} L_{t}\right) b-C_{1}\left(1-2 C_{2}\right) b^{b} L_{t} b= \\
& C_{3} L_{t}{ }^{b}
\end{aligned}
$$

La conclusión sería entonces, que bajo las hipótesis mencionadas, ambos índices de condición podrían considerarse proporcionales. Estas relaciones pueden extenderse fácilmente a los promedios de las muestras para concluir que $\overline{\mathrm{I}}=\mathrm{KP}$ std .

Las dos hipótesis que el autor considera fundamentales en este análisis son:

1) La forma exterior del bivalvo no cambia durante el crecimiento.

2) El espesor promedio de las valvas está linealmente relacionado con la longitud.

En realidad, ambas hipótesis se pueden resumir en la presuposición de crecimiento isométrico entre las variables largo, ancho, alto y espesor de valvas.

Para probar la validez. de la primera hipótesis en el caso de la almeja pismo, se analizó una muestra de 196 organismos, colectada en Playa San Ramón en agosto de 1979 , incluyendo todo el rango poblacional de longitudes. Los coeficientes paramétricos de correlación estimados para las relaciones largo-alto, largo-ancho y alto-ancho, son $0.999(\mathrm{P}<0.001), 0.995(\mathrm{P}<0.001)$ y $0.995(\mathrm{P}<0.001)$ respectivamente.

Por otro lado, en el caso de la segunda hipótesis, se analizó una submuestra de captura comercial colectada en octubre de 1979 , y se encontró un coeficiente de correlación de $0.500(P<0.001)$.

Entonces, existen evidencias poderosas a favor del uso del peso estándar en esta especie, como una medida de condición relacionada con el índice de Baird (1958).

La correcta comparación entre pesos estándar de diferentes muestras correspon- dientes a períodos distintos, requiere la presuposición de la constancia de las relaciones entre las dimensiones descritas, así como en la densidad de la carne. Aunque en este trabajo no se aportan evidencias al respecto, la experiencia de haber observado, medido y pesado cientos de almejas durante unos 3 años de estudio, nos indica que podemos confiar en esa consideración.

En resumen, podemos decir que el peso estándar es una alternativa para medir condición en bivalvos, con crecimiento isométrico, cuando se tienen sólo datos de longitud y peso de la carne de muestras de capturas comerciales, lo cual puede significar la optimización de la información generada en muchos estudios pesqueros. El trabajo estadístico es rclativamente sencillo con cualquier calculadora o computadora, y posiblemente la precisión al determinar pesos cs mayor que al determinar volúmenes. Por otro lado, se ha demostrado que el indice de Baird (1958) es dependiente de la longitud, y con el peso estándar se logra ajustar ese efecto a un valor puntual.

Otro índice de condición sugerido por Hickman e Illingworth (1980) para mejillones, es la proporción del peso de la carne respecto al peso total; sin embargo es dudable su aplicación en bivalvos de conchas gruesas como la almeja pismo. Para dar una idea de ésto, un organismo estándar de 420 y 70 gr de peso total y de carne respectivamente, que disminuya $10 \mathrm{gr}$ en el segundo valor, es decir un $14 \%$, reflejaría tan sólo un decremento del $2 \%$ en la proporción peso carne/peso total. Esos mismos autores muestran que, en su caso, existe una correlación significativa entre el índice de condición de Baird y otros índices basados en el peso seco y contenido de glucógeno.

\section{b) Relación del peso estándar con el estado reproductivo.}

El porcentaje de almejas a las que se les puede determinar el sexo, usado aquí como una medida cuantitativa del estado reproductivo, debe interpretarse con algunas reservas. El hecho que no se considere el estadio gametogénico, permite que una almeja se considere susceptible de determinar su se- 
xo desde el momento que presenta gametas reconocibles. De modo que una muestra con un $100 \%$ de P.A.S., no implica necesariamente el mismo porcentaje de almejas maduras.

Similarmente, en un organismo recién desovado pero en estado incompleto de reabsorción, su sexo podrá ser determinado fácilmente. Por este motivo una población muestreada poco antes y poco después del desove, podrá no revelar diferencias importantes en su P.A.S., a pesar de la importancia del evento.

Entre los muestreos de agosto y octubre de 1978, así como entre los de octubre y diciembre de 1979, se observó cualitativamente un descenso brusco del volumen gonadal que sc pucde intcrpretar como desove masivo. Las gónadas de mas del $80 \%$ de los organismos colectados en octubre de 1978 y diciembre de 1979 , se encontraban totalmente flácidas; mientras que en los muestreos anteriores de cada ciclo, incluían únicamente almejas con gónadas turgentes. Como se puede apreciar en la Figura 4a, en esos períodos se observan los mayores decrementos del peso estándar, que se pueden asociar entonces con los desoves masivos de cada ciclo. Posiblemente el decremento de septiembre de 1979 indique la presencia de desoves parciales.

Contrariamente a lo esperado, el P.A.S. permanece alto en las 4 muestras que siguieron al desove masivo de 1978, sugiriendo una lenta reabsorción de gametas, en comparación al ciclo de 1979 (Figura 4b). El coeficiente de correlación de Spearman, considerando el total de 20 parejas disponibles, aumentó de $0.499(\mathrm{P}<0.05)$ a $0.741(P<0.01)$, si se eliminan las 4 muestras mencionadas, dando una idea del efecto negativo que éstas producen en la correlación (Tabla 5).

Las otras dos discordancias importantes entre $P$ std y P.A.S., que se observan en los meses de febrero de 1979 y marzo de 1980 (Figura 4), coinciden en el primer caso, con los valores máximos de contenido de agua y cenizas de la Figura 6 . El porcentaje de aumento de ambos constituyentes en conjunto de diciembre de 1978 a febrero de
1980 , es de alrededor del $5 \%$, valor que si se aplica al peso estándar de febrero lo coloca dentro de los límites de confianza del mes siguiente. Adicionalmente, ambas discordancias ocurren un mes después de los volúmenes máximos de precipitación en cada ciclo (Figura 5).

Estas consideraciones sugieren la hipótesis de que los máximos de precipitación, podrían influir significativamente en el peso estándar, posiblemente a través de respuestas osmóticas y aumento de sedimentos en el tracto digestivo, ya que en los meses de precipitación máxima, se forma un arroyo de importancia que desemboca en Playa San Ramón (Searcy, 1982).

La adición de la variable volumen de precipitación (con desfase de un mes) a la correlación simple entre $\mathbf{P}$ std y P.A.S., producc un aumento significativo $(\mathrm{P}<0.05) \mathrm{cn}$ el coeficiente de determinación $(\rho 2)$ del 24.9 al $49.1 \%$ para el caso $n=20$. Cuando se eliminan los datos de las 4 muestras posteriores al desove $(n=16)$, el incremento de 54.9 a $56.6 \%$ no es significativo, debido posiblemente al aumento de la validez del P.A.S. como predictor (Tabla 5).

Sin embargo, parece ser que la inclusión de la nueva variable modifica los resultados en en sentido esperado; pero debido a otro efecto distinto al que se pretendía examinar.

El volumen de precipitación muestra una correlación negativa significativa tanto con el peso estándar como con el P.A.S. (Tabla 5), lo cual se podría explicar mediante la relación con otro factor o conjunto de factores que influyan sobre estas variables. Por ejemplo, el decremento de temperatura ambiental podría asociarse con volúmenes altos de precipitación; y a su vez influir en el descenso de la temperatura del agua, y ésta asociarse con un estadio reproductivo inactivo. Si este fuera el caso, el efecto de la lluvia sobre el peso estándar, que sería positivo en los meses de mayor precipitación, quedaría enmascarado en la correlación negativa persistente en la generalidad de los meses del año. 
Seria interesante la utilización de información sobre salinidad ambiental y sedimentos en suspensión, así como de presión osmótica interna, para reunir mas evidencias como prueba de la hipótesis señalada; sin embargo, no fue posible colectarla en este trabajo.

Continuar en este análisis multivariado, requiere de la inclusión de más variables y posiblemente de otros métodos estadísticos como análisis de ruta (Sokal y Rohlf, 1981), que están fuera de los límites de este trabajo.

Otra variable interesante de considerar, por ejemplo, sería algún índice de la condición nutritiva como el usado por Hines (1979) en mejillones. Hasta el momento no existe información suficiente para evaluar el efecto de este factor, posiblemente de importancia, considerándose que podría mostrar algún tipo de fluctuación cíclica relacionada con la disponibilidad de alimento, que afecte así la relación general entre peso estándar y actividad reproductiva. Es sintomático, sin embargo, que mas del $50 \%$ de la variación en el $\mathbf{P}$ std sea explicado con la sola consideración del porcentaje de almejas a las que se les puede determinar el sexo que, como se mencionó, no es el mejor índice que podemos usar para cuantificar la actividad reproductiva.

\section{CONCLUSIONES}

1.- El peso estándar puede ser una alternativa adecuada para medir condición en bivalvos, utilizando datos de longitud y peso de la carne, siempre y cuando se pueda asumir un crecimiento isométrico, como sucede con la especie aquí estudiada.

2.- Este índice parece reflejar de una manera general, los cambios de estado reproductivo de la almeja pismo en Playa San Ramón, B.C., siendo particularmente útil en la detección de desoves. Sin embargo, los volúmenes máximos de precipitación pluvial pueden tener, un efecto indirecto adverso a esta relación.

3.- La época de desove masivo de la especie en la zona de estudio, ocurrió entre agosto y octubre de 1978, y entre octubre y diciembre de 1979.

\section{LITERATURA CITADA}

BAIRD, R. H. 1958. Measurement of condition in mussels and oysters. J. Cons. Perm. Explor. Mer. 23:249-257.

CONOVER, W. J. 1980. Practical Nonparametric Statistics. J. Wiley and sons. N.Y. 493 p.p.

GABBOTT, P.A. y A. J. M. Walker. 1971. Changes in the condition index and biochemical content of adult oysters (Ostrea edulis L.) mantained under hatchery conditions. $J$. Cons. int. Explor. Mer. 34 (1): 99-106.

HICKMAN, R.W. and J. llingworth, 1980. Condition cycle of the green-lipped mussel Perna canaliculus in New Zealand. Mar. Biol. 60:27-38.

HINES, A. H. 1979. Effects of a thermal discharge on reproductive cycles in Mytilus edulis and Mytilus californianus (Mollusca, Bivalvia). Fish Bull. 77 (2): 498-503.

MACIAS, V., E. Gastélum y G. Leyva. 1979. Informe de avance del programa de bioquímica p.p. 145-162 en: Informe anual del Proyecto Bivalvos de Baja California: Sección Almeja Pismo. I.I.O. Ensenada, B. C., S.E.P., México, D.F.

SANTLAGO, R. y P. Rojas. 1982. Determinación del ciclo reproductivo del mej illón Mytilus californianus en el Ejido Eréndira, Baja California. Tesis de Licenciatura. Escuela Superior de Ciencias Marinas, UABC, Ensenada, B. C. $46 \mathrm{pp}$.

SEARCY, R. 1982. Evaluación preliminar sobre la pesquería de la almeja pismo Tivela stultorum (Mawe, 1823) en Playa San Ramón, B. C.: I Capturas Comerciales. Tesis de Licenciatura. Escuela Superior de Ciencias Marinas, UABC, Ensenada, B.C. 71 pp.

SNEDECOR, G.W. y W.G. Cochran. 1967. Statistical Methods, Iowa St. Univ. Press. Ames. $593 \mathrm{pp}$.

SOKAL, R.R. y F.J. Rohlf. 1981. Biometry. The principles and practice of statistics in biological research. W.H. Freeman and Co. San Francisco. 859 pp.

RICKER, W.E. 1973. Linear regressions in fishery research. J. Fish. Res. Bd. Can. 30:409434.

WALNE, P. R. 1970. The seasonal variation of meat and glyogen content of seven populations of oysters Ostrea edulis L. and review of the literature. Fishery Invest. Lond. Ser. 2, 26 (3): 35 pp. 